\title{
OPTICAL SPECTRA OF EXTRAGALACTIC RADIO SOURCES
}

\author{
E. Margaret Burbidge \\ (University of California, San Diego, Calif., U.S.A.)
}

\section{Introductory Remarks}

Extragalactic radio sources can be grouped spectroscopically into those that show absorption lines produced by a stellar population (e.g. the nearer radio galaxies, and some more distant ones, usually of D or E type) and those that show only lines (usually in emission) produced by low-density gas (e.g. QSO's and many N-type galaxies). If absorption lines from stars are present, one can estimate a minimum age from the stellar population, and perhaps a mass for the system from the stellar velocity dispersion. If no spectroscopic evidence on the stellar population is available (and this includes evidence from narrow-band filter photometry), then we have much less hold on the problems of age and evolutionary state. Absence of stellar absorption lines does not necessarily mean that stars are absent, but simply that their light, if present, does not contribute appreciably to the overall optical spectrum.

\section{QSO's: Emission-Line Spectra}

Forbidden and permitted lines are seen, of the general type found in galactic planetary or gaseous nebulae. Usually the lines are broad; a range of widths is sometimes found even in one object. The relative strengths of the emission lines can vary from one QSO to another, but Osterbrock and Parker found that average relative line intensities in 9 QSO's agree quite well with the computed line strengths for a gas with electron density $N_{\mathrm{e}} \approx 3 \times 10^{6} \mathrm{~cm}^{-3}$ and electron temperature $T_{\mathrm{e}} \approx 15000^{\circ} \mathrm{K}$, and 'solar-neighborhood' relative abundances. $3 \mathrm{C} 273$ has a higher electron density than this, but an upper limit is set by the presence of [O III] in its spectrum.

A single-fluid model with uniform $N_{\mathrm{e}}, T_{\mathrm{e}}$ has been shown, however, not to explain the observations as well as, e.g., a stratified model such as that proposed by Burbidge, Burbidge, Hoyle, and Lynds, or a model with denser filaments embedded in a more tenuous medium (first proposed by Schmidt; later, in more detail, by Woltjer). Perhaps a combination of both would be best. Woltjer's model has $N_{\mathrm{e}} \sim 10^{6}$ in the background and $N_{\mathrm{e}} \sim 10^{8}$ in the filaments. The importance of electron scattering was realized by Šklovskij and by Burbidge, Burbidge, Hoyle, and Lynds. The latter authors put it forward as an explanation for the emission-line widths, and particularly for the

Perek (ed.), Highlights of Astronomy, 356-360. (1.A.U. 
differences between permitted resonance lines, forbidden lines from higher excitation states, and forbidden lines from low states with fairly large cross-sections for collisional de-excitation.

The relative line strengths can be explained on the basis of abundance ratios of the elements similar to solar-neighborhood values, but, depending on the temperature, some underabundance of the elements heavier than $\mathrm{H}$ could also be present. The elements $\mathrm{H}, \mathrm{He}, \mathrm{C}, \mathrm{N}, \mathrm{O}, \mathrm{Ne}, \mathrm{Mg}, \mathrm{Si}, \mathrm{S}, \mathrm{Ar}, \mathrm{Fe}$, and possibly $\mathrm{Na}$ have been seen in emission. Tentative identifications of NaI by Wampler and Oke and OI by Véron may require more stratification in the model.

The emission-line red-shifts (101 objects) run up to $z=2 \cdot 22$; there are many around $z=2$, and it may be noted that the first 9 red-shifts published by Schmidt already included one - 3C 9 - with $z>2$. The red-shift-apparent-magnitude plot shows enormous scatter and a slope that is a factor of 2.5-3 steeper than the usual Hubble plot for normal or radio galaxies, facts which have led to their abandonment as tools for cosmological research. Some evidence has been found by Strittmatter, Faulkner, and Walmsley for a grouping in the sky of red-shifts according to the size of $z$.

Variations in the resonance feature MgII $2798 \AA$ have been observed by Burbidge and Burbidge, by Dibaj and Yesipov, and by Wampler in 3C 345. Šklovskij has explained this in terms of successive excitation and double ionization of moving clouds by bursts of electrons. On the other hand, the Ly- $\alpha$ and CIV lines in 3C 446 were found by Sandage, Strittmatter, and Westphal to fade when the object brightened by $3 \mathrm{mag}$. in a fashion consistent with the hypothesis that the lines remained at constant absolute flux while the continuum flux brightened. In July 1967, I found the lines to be plainly visible again at their former wavelengths; the object had faded to about 17.5 mag.

A correlation between UBV colors and red-shifts, discovered by McCrea and by Kardašev and Komberg, has been explained by Strittmatter and Burbidge as being due to the effect of the strongest emission lines superposed on a simple smooth underlying energy distribution in the continuum. Several other groups have recently done similar calculations and confirmed the results of Strittmatter and Burbidge.

\section{QSO's: Absorption-Line Spectra}

By May 1967, about 20 QSO's were known to have absorption lines in their spectra. The published identifications are mostly with resonance lines from the ground states of the cosmically abundant elements, though a few identifications that do not fall into this category have been proposed. Most of the QSO's with absorption lines have large emission-line red-shifts; of the 14 QSO's which have $z_{\mathrm{em}}>1 \cdot 9,10$ show ab. sorption, while of the remaining 87 with $z_{\mathrm{cm}}<1 \cdot 9$, only a further 10 show absorption. This cannot be wholly due to a preponderance of resonance lines in the region 1200 $1600 \AA$ among the relevant stages of ionization of the light elements, because Mg II $2796,2803 \AA$ and SiII $1808,1817 \AA$ are resonance lines that should appear in QSO's 
with smaller red-shifts. MgII absorption has actually been seen in PKS $0812+02$, 1229-02, and 1510-08.

The first object found to have many absorption lines was 3C 191; its lines were easily identified by Lynds, the Burbidges, and Stockton with Ly- $\alpha$, CII, CIv, Nv, SiII, SiIII, Sirv, and Sir transitions at $z_{\mathrm{abs}}=1.95$. A slight shortward shift of the absorption relative to the emission lines was interpreted as being due to an expanding shell of gas around the source of continuum radiation. The absorbing gas cannot be intergalactic, according to Bahcall, because excited fine-structure states of the ground level are populated. The absorption lines are narrow and deep, being only about $3 \AA$ wide in the rest frame. Bahcall, Sargent, and Schmidt found a C/Si abundance ratio of 3.5 , as compared with the solar-neighborhood value of 13 , but this needs further examination because of saturation curve-of-growth effects.

G. Burbidge noticed a preponderance of values of $z_{\text {abs }}$ near 1.95.3C 191 is one of these cases; PKS 0119-04 is another (it has $z_{\mathrm{abs}}$ slightly greater than $z_{\mathrm{em}}$, which means that, if $z_{\text {abs }}$ arises in a moving shell, the shell must be falling in towards the QSO continuum source and not expanding). Three of the objects with $z_{\text {abs }} \approx 1.95$ have two simultaneously present red-shifts; these are PKS 1116+12 (Bahcall, Peterson, and Schmidt), Ton 1530 (Hiltner, Cowley, and Schild; Lynds), and PKS 0237-23 (Arp, Bolton, and Kinman; Burbidge; Greenstein and Schmidt). The latter has $\Delta z_{\mathrm{abs}}=0.25$, corresponding to $\Delta v=0 \cdot 08 c$ if the shifts are Doppler. It has exceedingly narrow lines ( $\leqslant 1 \AA$ in the rest frame) and many unidentified lines. Other objects are PHL 938 (discovered by Kinman), PHL 5200 (found by Lynds to have a strange spectrum with wide absorption bands adjacent to broad emissions, like a supernova) and PHL 1127 (a radio-quiet QSO studied by Sandage and Luyten).

The conclusion is that absorption lines either have $z_{\mathrm{abs}}$ close to $z_{\mathrm{em}}$, or $z_{\mathrm{abs}} \approx 1.95$. This suggest a non-Doppler interpretation of the red-shift; Hoyle and Fowler (1967) put forward a model in which a gravitational red-shift is produced in a deep potential well in a configuration of collapsed objects with a pool of gas at the centre. A balance between radiation pressure outwards and gravitational attraction inwards, e.g., might maintain a stable thin shell around a continuum source.

PKS 0237-23 remains the most puzzling object. Arp et al. suggested identifications which would require abundances in heavier elements that are decidedly higher than the solar-neighborhood values. More work needs to be done on the identifications.

\section{Radio-Galaxy Spectra}

We return to the distinction made in Section 1 between objects with a spectroscopically detectable stellar population and those without. There is time to make only a few brief points:

(1) D and E type galaxies with absorption-line spectra of K-giant type (e.g. Vir A, Cen A, For A, and others more distant) must be $\sim 10^{10}$ years old. This fact, and the 
time-scale for relaxation of stellar systems, means that one must be wary of postulating that certain objects can evolve into other kinds, by considering the radio properties alone. The optical properties are paramount in considerations of age and evolutionary stages.

(2) Schmidt, in his study of 35 radio galaxies, noted that the absence of absorption features in the continuum is correlated with the strength of the emission lines. Presumably in certain strong radio galaxies the presence of many emission lines of higher stages of ionization and excitation (i.e. lines other than $\mathrm{H} \alpha,[\mathrm{O} I \mathrm{I}]$, and $[\mathrm{N}$ II] which are commonly present in normal galaxies) goes with the presence of continuum radiation that tends to swamp that coming from stars.

(3) There is a strong correlation between red-shift and the presence of strong emission lines, shown by the following tabulation:

For $z<.05,8$ out of 44 objects have 'strong emission' For $\cdot 05<z<\cdot 10,13$ out of 21 objects have 'strong emission' For $\cdot 10<z<\cdot 20,10$ out of 13 objects have 'strong emission' For $z>20,9$ out of 10 objects have 'strong emission'.

This is presumably a selection effect (one would like to know for how many faint galaxies attempts to measure red-shifts were made but failed because no strong emission lines were present).

(4) K-corrections calculated from a standard elliptical galaxy and applicable for $\mathrm{D}$ and $\mathrm{E}$ galaxies are dangerous to use for $\mathrm{N}$-type galaxies where an additional UV continuum may be present. Possibly a Seyfert nucleus like that of NGC 1068 would be best, or one of the nearby Zwicky compact galaxies; one of the latter is the best example of a non-radio $\mathrm{N}$-type galaxy that $\mathrm{I}$ have seen.

(5) MgII $2798 \AA$ should have appeared at $4090 \AA$ in 3 C 295 , but was not seen on Minkowski's spectra. It should also have appeared in 3C 109, with twice the red-shift of $3 \mathrm{C} 273$. This seems to indicate something different in the physical conditions in the two types of objects, even though 3C 109 has an emission-line spectrum otherwise rather similar to those of QSO's.

\section{Concluding Remarks}

The biggest spectroscopic problem posed by the QSO's at the moment is that of the absorption lines. A 'standard' red-shift of 1.95 raises again the question of gravitational red-shifts. PKS 0237-23 needs more work; there may be additional discrete red-shifts besides the two found, and there may be unusual element abundances.

\section{DISCUSSION}

Arp: The identification of the strongest absorption lines in PKS 0237-23 as due to Ti has been confirmed by spectroscopic observations with high resolution. 
Oke: There has been no detectable variation of the emission lines in $3 \mathrm{C} 446$ during the past year. Bidelman: When Fath measured the spectrum of NGC 1068 in 1908 with the Crossley telescope it required a $13 \frac{1}{2}$ hour exposure spread over 4 nights.

Arp: A direct photograph of M 87 (Virgo A) with an $\mathrm{H} \alpha$ interference filter has revealed a counterjet extending $40^{\prime \prime}$ arc from the nucleus in a direction opposite to that of the well-known jet. There are fainter extensions out to $127^{\prime \prime}$ arc. A spectrum shows the [O $\left.\mathrm{Ir}\right] 3727 \AA$ line as well as $\mathrm{H} \alpha$, in contrast to the spectrum of the original jet which is a blue continuum. If the distribution of galaxies in the neighbourhood of $\mathrm{M} 87$ is plotted they are found to lie along the line of the jet and counter-jet. There is a similar abnormal distribution of galaxies near the radio source Centaurus A (NGC 5128). (Ref. Astrophys. Lett., 1, 1, 1967.)

Kenderdine: The radio map of M 87 obtained with the 1-mile telescope at Cambridge shows the source extended along the line of the jet and counter-jet to about $30^{\prime \prime}$ arc on either side of the nucleus. This suggests that possibly Virgo A is like many other double sources with two clouds of plasma ejected from the nucleus, but that in this case we can see these clouds optically.

Fomalont: Observations at Cal. Tech. show that M 87 has a large radio halo about $6^{\prime}$ arc in diameter, as do some of the Seyfert galaxies and perhaps 3C 231 (M 82). 